\title{
OTIONOMTCS
}

Revista de economía, empresa y sociedad

Dossier «Economía social y solidaria: experiencias y retos»

VENTAJAS Y DESVENTAJAS DE LOS DIFERENTES MODELOS

\section{Monedas sociales y complementarias (MSC)}

\section{Yasuyuki Hirota}

Investigador de la Universitat de València

RESUMEN En las últimas décadas han surgido experiencias de monedas sociales y complementarias (MSC), medios de intercambio distintos del dinero de curso legal, con el fin de impulsar transacciones dentro del mercado. Su uso se justifica desde el punto de vista de la propia definición del dinero como acuerdo o ley dentro de una comunidad. Las MSC se clasifican en seis categorías distintas: las respaldadas con monedas oficiales (que optimizan la circulación de la moneda oficial al retenerla); las respaldadas con otros bienes y/o servicios (que inyectan liquidez en la comunidad); las emitidas por la autoridad pública (que circulan ampliamente por ser válidas en el pago de impuestos); las de confianza mutua (cuyos socios tienen saldos positivos o negativos, como derecho a pedir el valor equivalente de bienes o servicios, o como obligación a ofrecerlo); las emitidas como crédito bancario (que tienen efectos contracíclicos y permiten actividades económicas estables), y las FIAT (que nacen sin ningún respaldo y necesitan ser gestionadas cautelosamente para evitar acumulaciones que lleven a la hiperinflación). Es necesario estudiar las ventajas y desventajas de cada modelo antes de elegir el más apropiado.

PALABRAS CLAVE LETS; bancos del tiempo; monedas locales; monedas sociales; monedas complementarias

\section{Social and Complementary Currencies and timebanks}

\begin{abstract}
In recent decades, different manifestations of Social and Complementary Currencies (SCCs) have emerged. SCCs are exchange mechanisms that offer an alernative to legal tender, aiming to stimulate trade within a circle. Their use is justified by the very definition of money as an agreement or law made by the community. Such currencies can be divided into six categories: currencies backed by official currencies to optimize the circulation of the legal tender by retaining it; currencies backed by other goods and/or services to inject liquidity into the community; currencies issued by the public authority that are circulated extensively because of their usefulness for paying taxes; mutual credit systems where members' positive or negative balances equate to the right to ask for the equivalent value of goods and/or services or the duty to provide them, respectively; SCCs issued as bank credit, with counter-cyclical effects to stabilize economic activities; and Fiat SCCs, which come into being without collateral, and need to be carefully managed to avoid accumulations in some businesses or overissue leading to hyperinflation. Each model's advantages and disadvantages must be studied carefully to decide which is most appropriate.
\end{abstract}

KEYWORDS LETS; timebank; local currencies; social and complementary currencies 


\section{Introducción}

Las monedas sociales y complementarias (MSC) son herramientas para facilitar transacciones dentro de un determinado colectivo sin usar monedas de curso legal (euro, dólar estadounidense, libra esterlina, etc.). Se han desarrollado varios modelos, por ejemplo, los LETS y los bancos de tiempo, que funcionan en distintos países: Chiemgauer en Baviera (Alemania), Bristol Pound en Bristol (Reino Unido), Eusko en el País Vasco francés, La Turuta en Vilanova i La Geltrú (Barcelona), Puma en Sevilla, Banco Palmas en Brasil, clubes de trueque en Argentina, etc. Satisfacen diferentes necesidades socioeconómicas que no están cubiertas por el sistema monetario convencional.

En este artículo se presentan algunos de los fallos estructurales que impiden una operación sostenible del sistema monetario, algunos de los cuales son causados por la propia creación monetaria como crédito bancario que exige tasas de interés compuesto. A continuación se trata el concepto de MSC y se dan pinceladas sobre diferentes experiencias categorizadas en función del respaldo para su emisión. Por último, se comparan los diferentes modelos con el fin de mostrar sus propias características.

\section{Cinco fallos estructurales del sistema monetario}

Lietaer y otros (2012) mencionan los cinco aspectos siguientes acerca del sistema monetario actual, que funcionan en contra de la construcción de una sociedad sostenible:

1. Tendencia procíclica de creación y flujo monetarios. La banca privada es el mayor emisor de dinero como consecuencia de la creación crediticia. Se crea el dinero cuando esta concede créditos a los prestatarios (Douthwaite, 1999). Pero esta concesión depende de la coyuntura económica: suele inyectarse demasiada masa monetaria durante la bonanza, para calentar aún más la economía, mientras que las instituciones financieras rechazan ofrecer créditos una vez que comienza el retroceso económico, agravando aún más la economía.

2. Cortoplacismo. Debido a las tasas de interés compuesto se descuentan los valores actuales de las riquezas que se formarán en el futuro. Así, por ejemplo, al suponer que las tasas son del $5 \%$ al año, un depósito actual de $1.000 €$ serán unos $1.629 €\left(1.000 \times 1,05^{10}\right)$ dentro de 10 años. Por esta misma lógica, los activos que valdrán $1.000 €$ en 10 años valen actualmente solo unos $614 €\left(1.000 / 1,05^{10}\right)$. Por tanto los proyectos a largo plazo son poco atractivos para los inversores, mientras que les entusiasman otros proyectos que rinden a corto plazo.

3. Presión al crecimiento permanente. Otro efecto dañino de las tasas de interés en el desarrollo sostenible es la exigencia de un crecimiento cada vez más acelerado, puesto que la tasa de interés de los años anteriores también se convierte en principal. Esta presión obliga a que los actores económicos que toman préstamos prioricen su devolución, muy a menudo en detrimento del medio ambiente y/o de los trabajadores.

4. Concentración, sin misericordia, de la riqueza. Las tasas de interés funcionan para redistribuir la riqueza a favor de los pocos ricos, que tienen diversas formas de obtener rentas, mientras que la mayoría de la población paga más que recibe. Incluso aquellos sin deuda tienen que pagar esta tasa de interés como parte del precio de cada producto y/o servicio que consumen.

5. Devaluación del capital social (cohesión social). La competencia, promovida por el sistema monetario vigente, debilita las relaciones humanas.

Es muy relevante destacar que los fallos 1, 2 y 3 están vinculados con el hecho de que la mayoría de la masa monetaria está creada por la banca privada cuando concede créditos a los prestatarios para fines comerciales, exigiéndoles no solo devolver el principal, sino también las tasas de interés. Por tanto, «la masa monetaria de la economía real depende totalmente de la decisión del sector bancario sobre sus préstamos» (Dyson y otros, 
2011), mientras que la economía necesita cada vez más liquidez para poder pagar sus deudas. Este esquema funciona bastante bien cuando crece la economía y la banca ve más rentabilidad en este negocio, pero comienza a demostrar sus defectos estructurales tan pronto como se inicia el retroceso económico.

\section{Definición de MSC}

Uno de los hechos más curiosos es que, en comparación con los diferentes usos del dinero, se sabe muy poco sobre la naturaleza del mismo. Lietaer (2001) lo define como «un acuerdo dentro de una comunidad de usar algo como medio de intercambio», una definición similar a la de :Aristóteles: "No existe por naturaleza sino por ley» (Ética nicomáquea). Pero allí se añade el concepto de "comunidad» para destacar que cada moneda es propia de cada colectivo, que tiene la libertad de crear y usar su propio medio de intercambio siempre y cuando le sea apropiado. Un ejemplo serían las millas de las aerolíneas, aunque estos programas fueron creados por las propias aerolíneas como una herramienta de marketing.

El término MSC es la combinación de dos expresiones: monedas sociales y monedas complementarias. La primera fue creada por Primavera (1999) para destacar los «efectos de inclusión social» que se observaban en las prácticas de los clubes de trueque en Argentina, aunque la expresión se difundió luego por otros países en contextos socioeconómicos distintos al país rioplatense. La segunda fue acuñada por Bernard Lietaer en 1998 en un informe entregado a la Comisión Europea, en el que destacaba el papel de estas monedas a la hora de fomentar la cohesión social, mientras que la moneda convencional sirve para fortalecer el capital financiero y el físico (infraestructura) (Rizzo, 2003).

\section{Clasificación de las MSC}

Existen miles de MSC que pueden clasificarse en las seis categorías ya mencionadas: MSC respaldadas con dinero oficial, MSC respaldadas con otros bienes y/o servicios, MSC emitidas por entidades públicas, MSC basadas en las confianza mutua, MSC emitidas como crédito y MSC FIAT (Wild, 2011). Veamos, a continuación, cómo funciona cada modalidad en la práctica.

\subsection{MSC respaldadas con moneda oficial}

Chiemgauer (Alemania), Bristol Pound (Reino Unido), Berkshares (Estados Unidos), SOL-Violette y Eusko (Francia), entre otras divisas, pertenecen a esta categoría. Esta tipología de MSC se emite y circula en base al depósito de moneda oficial. Ciertos usuarios de este medio de intercambio pueden reembolsarla con el fin de pagar a sus proveedores externos, impuestos, etc., aunque en muchos casos se cobran comisiones por dicha operación con el fin de frenar la reconversión. Aunque es el modelo más cercano a la moneda convencional, está diseñado para retener la liquidez dentro de la zona por más tiempo, optimizando su efecto multiplicador.

Chiemgauer es la iniciativa más exitosa, no solo por su larga trayectoria histórica, sino también por su diseño sofisticado. Los consumidores eligen un proyecto social al darse de alta, al que donan un $3 \%$ cada vez que se realiza un cambio de euro a esa moneda. Los comercios que la aceptan pueden convertirlo a euro tras pagar el 5\% de comisión, o bien lo pueden gastar en otros comercios socios, escapándose de la comisión. En ocasiones se implementa la oxidación (Gesell, 1916), es decir, se cobra el 3\% de oxidación semestralmente (2\% trimestralmente hasta 2015) para desalentar el atesoramiento del dinero y estimular la circulación sin freno de los medios de intercambio. 


\subsection{MSC respaldadas con otros bienes y/o servicios}

El Banco de Horas Comunitario en Capilla del Monte, Córdoba (Argentina), y Gota Verde en Yoro (Honduras) corresponden a esta categoría. Así mismo, Terra, una propuesta de un comercial barter o permuta comercial a nivel planetario, también funcionaría de este modo una vez que fuera implementada.

La iniciativa argentina fue fundada en 1999 en la Cooperativa Escolar Olga Cossettini para permitir que los padres de los niños que estudiaban allí pudieran pagar parte de las mensualidades en esta MSC, que se emitía cuando cada uno depositaba productos o compromisos para ofrecer servicios. Ello hizo que las familias con orientaciones alternativas y con ingresos limitados en moneda oficial pudiesen matricular a sus hijos en el programa educativo que deseaban. Posteriormente participaron otras familias ajenas a la escuela que mostraron interés en usar este medio de intercambio (Caldano, 2007).

Gota Verde fue una experiencia iniciada gracias al apoyo de la fundación holandesa STRO, que impulsó un proyecto de producción de biodiésel para sustituir la importación de petróleo. Se emitía una MSC que era canjeable por aceite de jatropha (Puente Rodríguez, 2010; De Jongh y Nielsen, 2011).

Terra es una propuesta de Lietaer (2004) que se convertirá en una «moneda complementaria», de «emisión privada», que será «referenciada en comercio», "oxidable» y «resistente a la inflación», con el fin de superar los problemas en el comercio internacional de «falta de un estándar internacional de valor», «ampliación de los altibajos coyunturales», «punto muerto institucional» e «inacción política». Esta moneda será emitida como una iniciativa privada cuando las grandes corporaciones depositen materias primas de aceptación universal, tales como petróleo y trigo. Será reembolsable en estos depósitos, y se cobrará un $2 \%$ de oxidación anual en moneda oficial para mantener el sistema.

\subsection{MSC emitidas por entidades públicas}

Estas monedas suelen ser aceptadas por todos los actores económicos dado que, por su definición, tienen la ventaja de ser usadas como medio de pago de impuestos. Diferentes provincias argentinas, y hasta el propio Estado argentino, emitieron sus propios bonos y los pusieron en circulación durante la crisis de 2001-2002, conocidos como Patacón (Provincia de Buenos Aires) y Lecop (Estado argentino). Ello permitía complementar la falta de la masa del peso argentino, restringida por la convertibilidad (el Banco Central de la República Argentina necesitaba tener reserva en dólares para poder emitir este dinero de curso legal, es decir, el régimen monetario que existía era como patrón dólar en vez de patrón oro). Ello tenía como ventajas la reactivación de las economías provinciales en el período de aguda crisis y restricción, la estabilidad de precios y el freno a la fuga de capitales de los circuitos de acumulación y de la corrida del dólar (Sbatella, 2011). Soddy (1933) propone la emisión monetaria por parte del propio estado, con la obligación de reserva 100\% a la banca privada, para que se elimine el proceso de creación crediticia y que la autoridad monetaria gubernamental pueda inyectar una cantidad suficiente de liquidez en la economía nacional.

En los últimos años esta propuesta, conocida como «Chicago Plan», está siendo promovida por el movimiento social británico Positive Money y sus sucursales en diferentes países, además de contar con diferentes apoyos (Zarlenga, 2002; Stiglitz, 2003; Benes y Kumhof, 2013; Yamaguchi, 2013; Dyson, Jackson y Hodgson, 2014; Sigurjonsson, 2015).

\subsection{MSC basadas en la confianza mutua}

Los LETS y los bancos del tiempo, las dos corrientes más importantes de monedas sociales, pertenecen a esta corriente de MSC. Se trata de que todos los socios tienen una cuenta interna donde se registra cada transacción (uno aumenta su saldo mientras que otro lo reduce). Debido a esta estructura, es necesario que al menos alguien tenga el saldo negativo para que otro(s) socio(s) tenga(n) un sado positivo, si bien existe una diferencia fundamental entre la deuda en moneda oficial y el saldo negativo en estos sistemas de confianza mutua: no se cobran tasas de interés a los saldos negativos, impidiéndoles crecer exponencialmente. Los saldos positivos y negativos son «expectativas de la energía laboral» y «energía laboral disponible para cumplir obligaciones no hechas», respectivamente (Jackson, 1997). 
En algunos países los LETS están relacionados con la recuperación de los lazos tradicionales, tales como pumasi en Corea del Sur y Kaláka (ayuda mutua en el trabajo) en Hungría. En el país asiático algunas experiencias usan esta palabra autóctona para señalar la similitud entre esta moneda y una costumbre antigua que se practicaba sobre todo en el campo, mientras que en el centro de Europa se hace hincapié en la recuperación de tales relaciones humanas explícitas en la expresión (Hirota, 2012; North, 2004).

Della Peruta y Torre (2015) señalan que los LETS sirven para mantener la capacidad de los desempleados, mientras que Williams (1998), Gran (1998) e Ingleby (1998) hacen hincapié en el perfil particular de sus usuarios: verdes y/o izquierdistas y personas activas en la comunidad, con un alto nivel educativo y con ausencia de la clase obrera. Aldridge y otros (2003) cuestionan su utilidad de cara a la clase marginalizada. En España existen decenas de LETS que han nacido a partir de 2010, entre ellos: el Eco de la Cooperativa Integral Catalana, de las Ecoxarxes Catalanes y de la Xarxa 24 (Comunidad Valenciana), así como el Puma (Sevilla).

El Banco del Tiempo «beneficia a los socialmente excluidos por involucrarlos en el voluntariado mutuo y la participación comunitaria» (Seyfang, 2002), así como por crear una red de ayuda mutua sin involucrar el intercambio de bienes. Muchas iniciativas cuentan con el apoyo financiero del sector público y/o de fundaciones (por ejemplo, l'Accorderie en Québec y Francia) para cubrir los gastos necesarios para la operación. En España esta experiencia fue introducida por la asociación Salut i Familia y el Ayuntamiento de Barcelona, y luego se expandió a diferentes partes y dio lugar a centenares de iniciativas. Tal es el caso de Galicia, que cuenta con una ley que promueve bancos municipales (artículos 43 a 46 de la Ley Gallega 2/2007, de 28 de marzo, del trabajo en igualdad de las mujeres).

\subsection{MSC emitida como crédito bancario}

El Banco WIR, que funciona en Suiza desde 1934, es una cooperativa bancaria que concede crédito en su propia moneda (WIR) a tasas de interés más favorables para los prestatarios, reteniendo el poder adquisitivo dentro de la red de las empresas socias (pymes nacionales) y aumentando las transacciones entre ellas. El motivo de aceptar esta moneda es que los prestatarios necesitan devolverla. Stodder (2005) decribe diferentes efectos anticíclicos de esta moneda, que contribuyen a la estabilidad económica del país.

\subsection{MSC FIAT}

Pertenecen a esta categoría aquellas MSC emitidas a todos los socios sin ningún tipo de respaldo. Su funcionamiento se basa en la confianza de los usuarios en la moneda. Tal es el caso de Ithaca Hours, en el condado de Tompkins (Nueva York, EE. UU.), Calgary Dollars en Canadá y diferentes créditos usados en los clubes de trueque en Argentina hasta 2002. La experiencia estadounidense se enfrentó al problema de la acumulación de esta divisa en ciertos comercios, que no podían dispensarla (Papavasilou, 2008). Por otro lado, la experiencia sudamericana, que en su apogeo ayudó a millones de personas a sobrevivir, acabó perdiendo la confianza de los usuarios debido a la falta de control sobre la emisión, lo que inyectó demasiada masa monetaria, causando hiperinflación (Louge, 2005). La conclusión es que se necesita planificar muy bien cualquier proyecto de este tipo para facilitar la circulación continua y sin freno de la moneda, además de para controlar la masa monetaria evitando la subida de precios.

\section{Comparación de distintos modelos}

La soberanía monetaria y la aceptación tienen, en general, una relación opuesta. Las MSC de confianza mutua y las FIAT tienen el mayor grado de soberanía, y, en contrapartida, la aceptación de este medio de intercambio es muy limitada, puesto que lo aceptan solo los socios. En cuanto al grado de soberanía, a continuación se encuentran las respaldadas con bienes y/o servicios (donde hay que tener algo que depositar: este depósito aumenta la posibilidad de que sea aceptado), seguidas de las concedidas como crédito bancario y, por último, las respaldadas con dinero de curso legal (puesto que dependen totalmente de la moneda oficial). 
Normalmente los precios expresados en MSC son estables, aunque en el caso de las FIAT es necesario controlar la masa monetaria, ya que se corre el riesgo de generar hiperinflaciones. Todas las MSC, a excepción de las respaldadas con dinero de curso legal, son contracíclicas. Desde el punto de vista de la inclusión social, las monedas emitidas por las entidades públicas tendrán mayor potencial, dado que es posible destinarlas a la mejora de la calidad de vida de la clase más marginalizada, aunque emplearlas para otros fines es completamente posible. Esta misma potencialidad la presentan las monedas basadas en la confianza mutua y las FIAT. En cuanto a la fidelizacón de clientes, las basadas en la confianza mutua, las respaldadas en moneda oficial y las respaldadas con otros bienes y/o servicios suelen ser restringidas al uso local, aumentando la posibilidad de atraer clientes.

\section{Conclusiones}

Las monedas oficiales tienen diferentes fallos estructurales, tales como la tendencia procíclica de creación y flujo monetarios, el cortoplacismo, la presión al crecimiento permanente, la concentración inmisericorde de la riqueza y la devaluación del capital social (cohesión social). Todos ellos funcionan en contra de la sostenibilidad de nuestra sociedad. Es destacable el hecho de que los primeros tres fallos son fruto de la propia creación monetaria como crédito bancario, con tasas de interés compuesto a pagar. La propia inyección del medio de intercambio depende de la rentabilidad de la banca.

El dinero es simplemente una ley o un acuerdo dentro de una comunidad, de manera que se puede crear cualquier otro medio de intercambio siempre y cuando exista un consenso entre sus usuarios. Cada MSC funciona de un modo particular, según su respaldo. Las respaldadas con moneda oficial optimizan la circulación de la propia moneda oficial, mientras que las respaldadas con otros bienes y/o servicios aumentan la liquidez a base de riqueza en la comunidad, las emitidas por entidades públicas circulan dentro de la economía nacional, las basadas en la confianza mutua generan transacciones en ciertos grupos, las emitidas como crédito bancario estabilizan la economía por sus efectos contracíclicos y, finalmente, las FIAT necesitan una gestión apropiada. Cada modelo tiene sus propias ventajas y desventajas, dependiendo del puntos de vista. Por todo ello, es importante elegir siempre el más adecuado.

\section{Bibliografía}

ALDRIDGE, T. J.; PATTERSON, A.; TOOKE, J. (2003). «Trading Places: geography and the role of Local Exchange Trading Schemes in local sustainable development». En: Buckingham, S.; Theobald, K. (eds.). Local environmental sustainability. Cambridge: Woodhead.

BENES, J.; KUMHOF, M. (2013). The Chicago Plan Revisited. Washington, D.C.: International Monetary Fund. Disponible en línea: <http://www.lse.ac.uk/economics/newsEventsSeminars/files/MichaelKumhofpaper.pdf>.

CALDANO, M. (2007). «La experiencia de la Cooperativa Escolar y Banco de Horas Comunitario "Olga Cossettini"». En: Plasencia A.; Orzi, R. (comps.). Moneda social y mercados solidarios - Potencial emancipador y pedagógico de los sistemas monetarios alternativos (págs. 89-96). Buenos Aires: Ciccus.

DE JONGH, J.; NIELSEN, F. (2011). Lessons Learned: Jatropha for local development. Liverpool: FACT. Disponible en línea: <http://www.jatropha.pro/PDF\%20bestanden/2011-12_Jatropha_Lessons_Learned_final.pdf>.

DELLA PERUTA, M.; TORRE, D. (2015). «Virtual Social Currencies for Unemloyed People: Social Networks and Job Market Access». International Journal of Community Currency Research. Vol. 19, sección D, págs. 31-41. DOUTHWAITE, R. (1999). The Ecology of Money. Totnes, UK: Greenbooks.

DYSON, B. y otros (2011). Towards a twenty-first century banking and monetary system submission to the independent commission on banking. Londres: Positive Money. Disponible en línea: <http://www.positivemoney. org/wp-content/uploads/2010/11/NEF-Southampton-Positive-Money-ICB-Submission.pdf>. 
DYSON, B.; JACKSON, A.; HODGSON, G. (2014). Creating a Sovereign Monetary System. Londres: Positive Money. Disponible en línea: <http://positivemoney.org/wp-content/uploads/2014/07/Creating_a_Sovereign_Monetary_System_Web20130615.pdf>.

GESELL, S. (1916). Die natürliche Wirtschaftsordnung durch Freiland und Freigeld. Les Hauts Geneveys, Suiza: Selbstverlag.

GRAN, E. (1998). «Green Domination In Norwegian Letsystems: Catalyst For Growth Or Constraint On Development?». International Journal of Community Currency Research. Vol. 2.

HIROTA, Y. (2012). Monedas sociales y complementarias (MSCs): Experiencias, su papel en la economía social, estrategias, marketing y políticas públicas. Valencia: Universidad de Valencia. Disponible en línea: <http:// www.slideshare.net/mig76/monedas-sociales-y-complementarias-ms-cs-experiencias-su-papel-en-la-economa-social-estrategias-marketing-y-polticas-pblicas>.

INGLEBY, J. (1998). «Local Economic Trading Systems: Potentials for New Communities of Meaning: a brief exploration of eight LETSystems, with a focus on decision making». International Journal of Community Currency Research. Vol. 2.

JACKSON, M. (1997). «The Problem of Over-Accumulation: Examining and Theorising The Structural Form of LETS». International Journal of Community Currency Research. Vol. 1.

LIETAER, B. (2001). The Future of Money - Creating new wealth, work and a wiser world. Londres: Century.

LIETAER, B. y otros (2012). Money and Sustainability - The Missing Link. Devon, UK: Triarchy Press.

LOUGE, C. (2005). «Historia del trueque en Latinoamérica y la Argentina». En: Lietaer, B. El Futuro del Dinero. Buenos Aires: Longseller.

NORTH, P. (2004). «Kaláka and Kör: Green money and mutual aid in Hungary». International Journal of Community Currency Research. Vol. 8, págs. 24-28.

PAPAVASILOU, F. (2008). The Political Economy of Local Currency: Alternative Money, Alternative Development and Collective Action in the Age of Globalization. Atlanta: Emory University. Disponible en línea: <https://etd. library.emory.edu/file/view/pid/emory:15tfn/papavasiliou_dissertation.pdf>.

PRIMAVERA, H. (1999). «La moneda social de la Red Global de Trueque en Argentina: ¿Barajar y dar de nuevo en el juego social?». I Seminario Internacional «Globalisation of Financial Markets and its Effects on the Emerging Countries", Santiago de Chile, 29-31 de marzo 1999. Disponible en línea: <http://base.socioeco.org/docs/ doc-7262_es.pdf>.

PUENTE RODRÍGUEZ, D. (2010). Redesigning Genomics - Reconstructing Societies: Local Sustainable Biotechnological Developments. Ámsterdam: Vrije Universiteit. Disponible en línea: <http://dare.ubvu.vu.nl/handle/1871/15887>.

RIZZO, P. (2003). L'économie sociale et solidaire face aux expérimentations monétaires - Monnaies sociales et Monnaies multilatérales. París: L'Hermattan.

SEYFANG, G. (2002). "Tackling social exclusion with community currencies: learning from LETS to Time Banks». International Journal of Community Currency Research. Vol. 6.

SIGURJONSSON, F. (2015). Monetary Reform: a better monetary system for Iceland. Reykjavik. Disponible en línea: <http://www.forsaetisraduneyti.is/media/Skyrslur/monetary-reform.pdf>.

SODDY, F. (1933/1961). Wealth, Virtual Wealth and Debt. Londres: Omni Publications.

STIGLITZ, J. (2003). Deflation, Globalization and The NewParadigm of Monetary Economics. Disponible en línea: $<$ http://www.mof.go.jp/english/about_mof/councils/customs_foreign_exchange/gaic20030416.htm>.

STODDER, J. (2005). «Reciprocal Exchange Networks: Implications for Macroeconomic Stability». Disponible en línea: <http://www.lietaer.com/images/Stodder_Reciprocal_Exchange.pdf>.

WILD, L. (2011). El Dinero o la vida - Una guía práctica para la alquimia monetaria. Quito: Mayor Books.

WILLIAMS, C. C. (1997). «Local Exchange And Trading Systems (LETS) In Australia: A New Tool For Community Development?» International Journal of Community Currency Research. Vol. 1.

YAMAGUCHI, K. (2013). Money and Macroeconomic Dynamics - Accounting System Dynamics Approach. Osaka: Japan Futures Research Center.

ZARLENGA, S. (2002). The lost science of money - The Mythology of Money - They Story of Power. Valatie, Nueva York: American Monetary Institute. 


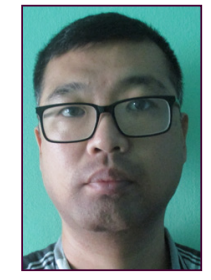

\section{Yasuyuki Hirota}

miguel@ineval.org

\section{Investigador de la Universitat de València}

Doctorando japonés de Economía Social en el Instituto Universitario de Investigación en Economía Social, Cooperativismo y Emprendimiento (IUDESCOOP) de la Universitat de València. A nivel personal sigue investigando y promoviendo prácticas de monedas sociales desde 1999 desde el punto de vista del marketing. Ha publicado tres libros en japonés sobre monedas y un cuarto libro sobre economía social y solidaria, que está por publicarse, además de ser cofundador del Instituto de la Moneda Social (http://www.monedasocial. org/) y escribir posts sobre el tema en el blog de El País (Lista de posts: http://www.monedasocial. org/?p=70) desde 2013.

Los textos publicados en esta revista están sujetas -salvo que se indique el contrario- a una licencia de Reconocimiento 3.0 España de Creative Commons. Podéis copiarlos, distribuirlos, comunicarlos públicamente y hacer obras derivadas siempre que reconozcáis los créditos de las obras (autoría, nombre de la revista, institución editora) de la manera especificada por los autores o por la revista. La licencia completa se puede consultar en http://creativecommons.org/licenses/by/3.0/es/deed.ca.

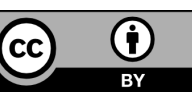

